\title{
Effect of anodal and cathodal microamperage direct current electrical stimulation on injury potential and wound size in guinea pigs
}

\author{
Gadamali Talebi, MSc; ${ }^{1}$ Giti Torkaman, PhD; ${ }^{*}$ Mohammad Firoozabadi, PhD; ${ }^{2}$ Shams Shariat, $\mathbf{P h D}^{3}$ \\ Departments of ${ }^{1}$ Physical Therapy and ${ }^{2}$ Medical Physics, Tarbiat Modares University, Tehran, Iran; ${ }^{3}$ Tehran Medical \\ Sciences University, Tehran, Iran
}

\begin{abstract}
Injury potential may have a regulatory role in the wound healing process, and exogenous electrical stimulation (ES) may mimic natural endogenous bioelectric current that can improve wound healing. Until now, the influence of externally applied ES on injury potential has not been demonstrated during the healing of acute wounds. Thirty-nine male guinea pigs were randomly divided into a control group (sham treatment) and two experimental groups: anodal and cathodal direct current (DC). A $2.5 \mathrm{~cm}$-long full-thickness skin incision was made on each animal's dorsal region. Differential skin surface potential was measured before and immediately after the injury and also through day 21 of the healing period; wound surface area (WSA) was also measured throughout the 21-day healing period. Immediately after injury, wound potential significantly increased in all three groups, reaching a maximum on day 1 for the control and cathodal groups and day 3 for the anodal group $(p<0.05)$, then decreasing through the healing period. Wound potential returned to preinjury levels by the end of the healing period in the anodal group only. By days 19 and 21, wound potential had decreased more for the anodal group than the control group $(p<0.05)$. By day 15 for the anodal group and day 17 for the cathodal group, WSA had decreased more compared with the control group $(p<0.05)$. Anodal microamperage DC ES is appropriate for improving the healing of acute skin wounds because it causes both the wound surface to close and the wound potential to return to preinjury levels faster.
\end{abstract}

Key words: anodal, cathodal, direct current, electrical stimulation, full-thickness skin wound, guinea pig, injury potential, microamperage, rehabilitation, wound healing, wound surface area.

\section{INTRODUCTION}

Skin wounds can be a major medical, social, and economic problem for patients [1]. Chronic ulcers (pressure sores, diabetic ulcers, venous stasis ulcers, nonhealing ulcers, etc.) are especially troublesome problems, with complex and not totally understood etiologies, that affect numerous patient populations. Studies on the acceleration of wound healing are very important for some specialists (rehabilitation medicine, dermatologists, surgeons, etc.). During the last several decades, many physical modalities, including ultraviolet radiation, electrical stimulation (ES), pulsed electromagnetic fields, low-energy laser, and ultrasound, have been used to promote wound healing [1-7]. Of all the physical modalities used to augment wound healing, the greatest amount of evidence supports the use of ES [1,8-12]. These studies evidenced two important findings. First, human or animal skin possesses endogenous electrical potentials such that the external skin surface is always electronegative with respect to inner skin layers [13-14].

Abbreviations: ANOVA $=$ analysis of variance, $\mathrm{DC}=$ direct current, DSSP = differential skin surface potential, \%DWP = percent decreased wound potential, $\%$ DWSA $=$ percent decreased wound surface area, ES = electrical stimulation, HVPC = high voltage pulse current, WSA = wound surface area.

*Address all correspondence to Giti Torkaman, PhD; Department of Physical Therapy, Tarbiat Modares University, Tehran, Iran; +98-21-88011001, ext 4509; fax: +98-2188006544. Email: torkamg@modares.ac.ir

DOI: 10.1682/JRRD.2007.05.0068 
Second, wound sites are positive compared with surrounding intact skin [15-17]. Because of potential differences between the injury site and the surrounding intact skin, a low-intensity direct current (DC) flows between them [1314]. In actuality, following tissue damage, an injury current is generated that is thought to trigger biological repair [14,18-19]. Based on these finding, researchers have proposed that external ES may help healing through the simulation and enhancement of natural bioelectric currents $[3,13,19-20]$. This theory is considered one of the most important mechanisms of the effectiveness of ES on wound healing [1,3]. However, the actual effect of ES on injury potential in acute wounds during the healing process has not been studied.

Skin potential has typically been studied through transcutaneous potential measurement (the potential difference between the outer skin surface and the inner skin layers), which is an invasive method [14-15,21-22]. An electrode must be inserted into the skin and another movable electrode placed on the skin. To measure the transcutaneous potential, researchers must puncture the epidermis [23]. In this method, the epidermal puncture will inevitably generate additional injury potential. Therefore, to evaluate wound potential during healing, we selected the differential skin surface potential (DSSP) measurement technique. In this method, two surface electrodes are placed at specific locations along with a single reference electrode that is common to all sites and we measure the surface potential difference between any two of the electrodes [24-26]. With the DSSP technique, we anticipate that factors including age, sex, and environmental variables, such as temperature and humidity, will affect both electrode sites similarly and therefore not result in a change in DSSP magnitude [23].

This study was designed to answer the following questions: Does externally applied ES influence wound potential? Does ES return wound potential to preinjury levels sooner? What is the relationship between changes in wound potential and wound surface area (WSA)? Which DC ES polarity has better effects on the return of wound potential to preinjury levels and wound closure? Does a valid and reliable relationship exist between differential potentials recorded from the skin surface and the progress of a skin lesion from injury to recovery? This study investigated the effects of anodal and cathodal microamperage DC ES on wound potential and WSA in guinea pigs.

\section{MATERIALS AND METHODS}

\section{Animals}

In this investigation, we used 39 healthy, male, adult, albino, Dunkin-Hartley guinea pigs (Pasteur Institute; Tehran, Iran) weighing 350 to 400 g. The animals were maintained in special cages according to the controlled conditions in the experimental guidelines of Tarbiat Modares University. Food was accessible to the animals without any limitation until 12 hours before the surgery. The study was approved by the Ethical Commission of Tarbiat Modares University.

\section{Differential Skin Surface Potential Measurement}

For the endogenous potential measurement, we used a custom-designed differential amplifier device (BPT Co; Tehran, Iran), with an input impedance of $10^{8} \Omega$ and a common mode rejection ratio of $100 \mathrm{db}$. To omit any alternative current, electromyography, or other interference signals, we used a $0.25 \mathrm{~Hz}$ low-pass filter. To amplify the very small skin potential signals, we set the gain of device at 10 . Predetermined voltages were generated by an instrument signal generator (model FG-330; Iwatsu, Japan) and measured with a custom-designed potentiometer to ensure electronic calibration. We observed that the custom-designed differential amplifier could read the predetermined voltages correctly. The measurement setup included the customdesigned differential amplifier instrument and a digital multimeter (Digital HiTester, model 3801; Hioki EE Corp, Japan) that digitally displayed the recorded differential potential. Initial measurements demonstrated that the recorded signals fluctuated greatly initially but achieved stability 15 minutes after electrode application. Therefore, each data acquisition session was 20 minutes long.

In four animals, the potential difference between the two skin sites was measured three times and continued for 4 days. Analysis of variance (ANOVA) showed no significant differences between the mean potentials on the different days. Therefore, the designed potentiometer is reliable for repeated measurements.

\section{Surgical Procedure}

After weighing them, we anesthetized the animals using a mixture of xylazine $(20 \mathrm{mg} / \mathrm{mL})$ and ketamine hydrochloride $(100 \mathrm{mg} / \mathrm{mL}$ ) (xylazine:ketamine ratio of $1: 8 \mathrm{cc}$ and dose of $1 \mathrm{cc} / \mathrm{kg}$ ) [27]. The hair on the middle of the back of each guinea pig was shaved and the area was cleaned with Betadine ${ }^{\circledR}$ antiseptic solution. Following the sterilization, we made a $2.5 \mathrm{~cm}$-long full-thickness incision 
at a distance of $1 \mathrm{~cm}$ from the spine on the right side of the paravertebral region (Figure 1).

\section{Treatment Protocol}

Thirty-nine guinea pigs were randomly divided into one control and two experimental groups. Each group included 13 animals. Treatment began 24 hours after injury. Electrodes were placed on sterile pads moistened with saline solution. An active treatment electrode was placed on the wound and a passive indifferent electrode on the opposite side of the paravertebral region and as far away as possible from the wound. In one of the experimental groups, the polarity of the treatment electrode was anodal throughout the 3-week healing period. In the other experimental group, the polarity of the treatment electrode was cathodal throughout the 3-week healing period. To prevent electrode displacement, we tied the electrodes tightly using straps. Using a stimulator device (BTL 5000 series; BTL Industries, Ltd, United Kingdom), we applied microamperage DC ES with an intensity of $600 \mathrm{~A}$, for $1 \mathrm{~h} /$ day, three times a week, for 3 weeks. These treatment parameters are used in many other studies [28-29]. In the control group, electrodes were similarly placed on the wound site but no current was applied.

\section{Measurements}

We measured the DSSP of the wound site relative to the adjacent intact skin at a distance of $2 \mathrm{~cm}$; the reference electrode was always placed distally on intact skin (Figure 2). The DSSP of the wound was measured before injury, immediately after injury, and once a day throughout the healing period. In each session, we measured the wound potential three times for 20 minutes each time.

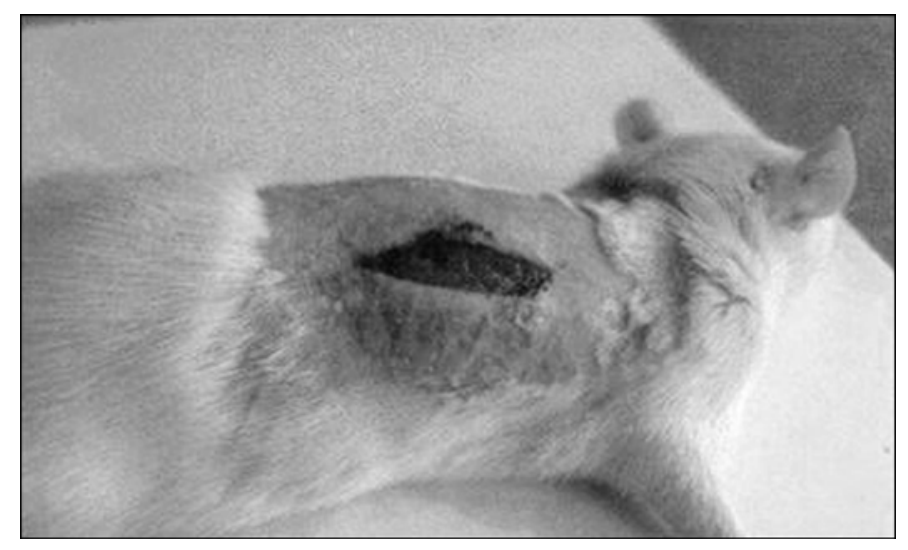

Figure 1.

Sample of full-thickness skin wound on guinea pig 1 day after incision.
Also, the WSA was measured daily throughout the healing period. The percent decreased WSA (\%DWSA) from the initial evaluation was calculated for each animal by

$\%$ DWSA $=[($ initial WSA - WSA on each day $) /$ initial WSA $] \times 100$

To improve the accuracy of these measurements, we anesthetized the animal and traced each wound three times. Tracing was performed with transparent plastic paper, and WSA was calculated by planimetry. We identified the tracings with code numbers to exclude observer bias.

In addition, the weekly healing rate (\%DWSA in each week) was calculated during the first, second, and third weeks (comparison of WSA on the first and seventh day of each week) by

$\%$ DWSA in week $=[($ WSA on 1st day in week -

WSA on 7th day in week $) /($ WSA on 1st day in week $\times 100)$.

\section{RESULTS}

\section{Changes in Injury Potential}

Before wounding, the mean \pm standard deviation DSSP was $-0.5 \pm 1.75 \mathrm{mV}$ for the control group, $0.09 \pm$ $0.82 \mathrm{mV}$ for the cathodal group, and $-0.42 \pm 1.15 \mathrm{mV}$ for the anodal group. Paired $t$-tests showed that in each group, the wound site differential potential (wound potential) increased significantly immediately after wounding $(p<0.05)$ (Figure 3). The maximum wound potential value was not obtained immediately after injury but occurred 1 day later in the control and cathodal groups and 3 days later in anodal group. After these maximum

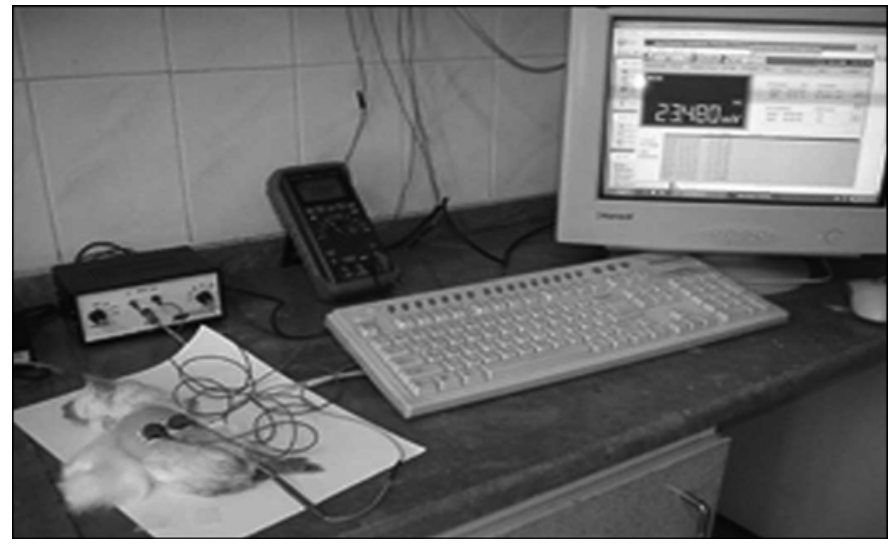

Figure 2.

Setup for measuring wound site differential potential. 


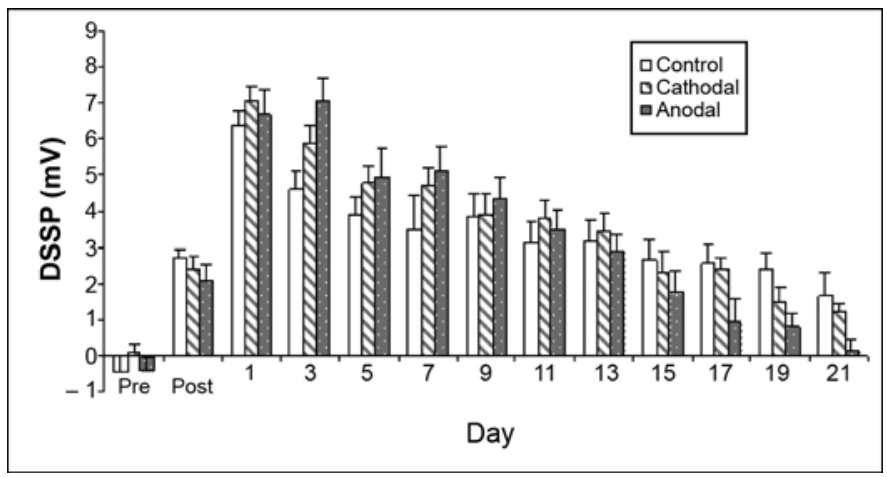

Figure 3.

Changes in differential skin surface potential (DSSP) of wound site for three different groups through healing period (day 21). Error bars represent standard error of the mean. Post = postinjury, Pre = preinjury.

levels, wound potential decreased progressively through the healing period in all the groups.

Also, ANOVA revealed no significant differences between groups in baseline potential before wounding $(p>0.05)$. The measured potential on days 19 and 21 was significantly lower in the anodal group compared with the control group. No significant differences were found between the cathodal and control groups for any testing day. Although the measured potential in the cathodal group was lower than the control group in the third week, this difference was not significant.

Paired $t$-tests for the anodal group found no significant differences between the mean potentials before wounding and on days 17,19 , or $21(p>0.05)$. For the control and cathodal groups, significant differences were found between the mean potentials before wounding and all days after injury $(p<0.05)$. Wound potential changes for the different groups are shown in Figure 3.

\section{Changes in Wound Surface Area}

Kolmogorov-Smirnov tests demonstrated that the WSA on the first day after injury had a normal distribution in all of the groups. No significant differences were found between the groups for WSA on the first day after injury ( $p>0.05)$. \%DWSA was significantly higher in the anodal group than control group on days 15, 17, 19, and 21 . Also, \%DWSA was significantly higher in the cathodal group than the control group on days 17, 19, and 21. Although \%DWSA in the anodal group was higher than the cathodal group in the third week, this difference was not significant $(p>0.05)$. WSA changes for the different groups are seen in Figure 4.

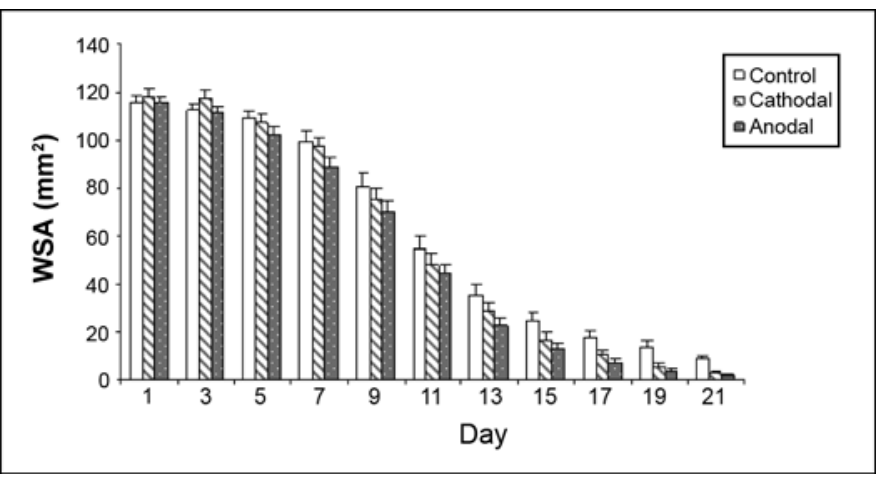

Figure 4.

Changes in wound surface area (WSA) for three different groups through healing period (day 21). Error bars represent standard error of the mean.

Comparison of \%DWSA during each week demonstrated that wound size decreased through the second week in each of the three groups (Table 1).

ANOVA revealed that \%DWSA in the anodal group was significantly greater than the control group by the third week $(p<0.05)$, but differences between the anodal and cathodal groups and between the cathodal and control groups were not significant $(p>0.05)$.

\section{Correlation Between Changes in Wound Potential and Wound Surface Area}

We found that the endogenous potential of the wound site decreased progressively as WSA decreased. After reaching a peak value, the endogenous potential of the wound site decreased in all the groups and approached baseline values. The greatest correlation between wound potential and WSA was observed for the anodal group $\left(R^{2}=0.94\right)$. Comparison of \%DWSA and percent decreased wound potential (\%DWP) from its peak value supports this finding (Table 2).

\section{DISCUSSION}

The overall pattern of changes in the endogenous potentials of injured skin was similar in control and experimental groups. After wounding, the endogenous potentials of injured skin were positive relative to the intact surrounding skin and reached their peak values 1 day (control and cathodal groups) or 3 days (anodal group) later. Afterward, the endogenous potentials of injured skin decreased with the progression of the healing 
Table 1.

Mean \pm standard deviation percent decreased wound surface area for three different groups through healing period (day 21).

\begin{tabular}{lccc}
\hline Group & $\begin{array}{c}\text { Through } \\
\text { 1st Week }\end{array}$ & $\begin{array}{c}\text { Through } \\
\text { 2nd Week }\end{array}$ & $\begin{array}{c}\text { Through } \\
\text { 3rd Week }\end{array}$ \\
\hline Control & $14.62 \pm 12.41$ & $74.89 \pm 11.08$ & $68.26 \pm 17.63$ \\
Cathodal & $17.10 \pm 9.80$ & $82.77 \pm 11.36$ & $83.25 \pm 23.56$ \\
Anodal & $23.08 \pm 9.89$ & $84.42 \pm 10.53$ & $90.30 \pm 12.24{ }^{*}$ \\
"Significantly different from control group, $p<0.05$. \\
\hline
\end{tabular}

Table 2.

Correlation between changes in percent decreased wound surface area (\%DWSA) and percent decreased wound potential (\%DWP) for three different groups.

\begin{tabular}{lccc}
\hline Group & $\begin{array}{c}\text { \%DWSA } \\
\text { Day 21 }\end{array}$ & $\begin{array}{c}\text { \%DWP Peak } \\
\text { to Day 21 }\end{array}$ & $\begin{array}{c}\text { Correlation } \\
\text { Between \%DWSA } \\
\text { and \%DWP }\left(\boldsymbol{R}^{\mathbf{2}}\right)^{*}\end{array}$ \\
\hline Control & 92.17 & 73.89 & 0.74 \\
Cathodal & 97.53 & 83.16 & 0.88 \\
Anodal & 98.52 & 98.44 & 0.94 \\
\hline *Signifint
\end{tabular}

*Significant correlation between \%DWSA and \%DWP for all groups, $p<0.05$.

process but remained positive relative to the intact surrounding skin throughout the 3-week healing period. Cunliffe-Barnes [16], Illingworth and Barker [17], and Jerinovi et al. [24] reported that endogenous electrical potentials of wounds remained positive compared with surrounding intact skin throughout the healing process. But Bur et al. [30], Becker [31], and Chang and Snellen [32] found that the charge of endogenous potentials can change during healing.

In the control group, \%DWSA was greater compared with \%DWP from its peak value. This finding indicates that skin closing in acute wounds under usual conditions (without externally applied current) occurs faster than wound potential returns to preinjury levels. Wound closure may occur faster for two reasons. First, closing of the wound surface may be a more important response for the body. Endogenous electrical currents may stop in infectious and chronic conditions and return to initial preinjury levels with delay [3]. Faster wound closure prevents wounds from becoming infected and chronic and, therefore, helps wound potential return to preinjury levels. The second reason may be the mechanisms that produce the basal skin potential. These mechanisms are multiple and complex and include epidermal membranes, "skin battery" (voltage across the epidermis), sweat glands, and some diffusion and active-pumping mecha- nisms [13-14,21-22,33]. As a result, the return of wound potential to preinjury levels is a more prolonged and complex process that occurs slower than wound closure.

In the cathodal group, \%DWSA was 97.53 percent and \%DWP from its peak value was 83.16 percent, but in the anodal group, the amount of wound closure and the amount of wound potential that returned to initial preinjury levels was the same (Table 2). Also, the greatest correlation between these two variables was observed in the anodal group. These findings demonstrate that anodal DC ES could return wound potential to preinjury levels sooner and reduce the gap between wound closure and return of wound potential to preinjury levels.

We found that the endogenous potentials of injured skin returned to preinjury levels by the end of the third week in the anodal group only. Also, the endogenous potentials of the injured skin in the anodal group decreased more by days 19 and 21 compared with the control group, whereas the differences between the two experimental groups were not significant. This finding also supports that anodal DC ES can return the endogenous potentials of injured skin to preinjury levels faster by precipitating endogenous bioelectric events of the wound.

Some authors have proposed that endogenous bioelectric currents of a wound may play an important role in attracting epithelial cells and, therefore, in closing the wound surface $[13,34]$. Injured skin possesses a positive charge compared with the surrounding intact skin, and epithelial cells may migrate toward this positive charge $[8,16]$. Based on these findings, anodal microamperage DC ES can improve wound closing by accelerating the endogenous bioelectric events of the wound and attracting the epithelial cells. Karba et al. reported that the endogenous electric conditions in the skin were closely approximated with external ES when the wound surface was covered with the positive stimulation electrode while the negative electrode was applied to the tissue surrounding the wound [9].

Comparison of \%DWSA in different groups demonstrated that anodal stimulation was more successful in closing the wound surface. Although both anodal and cathodal stimulation reduced the wound surface more than the sham treatment, in the anodal group, the significant reduction with respect to the control group occurred sooner. Additionally, \%DWSA was significantly higher in the anodal group compared with the control group through the end of the healing period, while the difference in \%DWSA between the cathodal and control groups was not significant. 
Alvarez et al. reported that positive polarized current caused more rapid epithelialization with respect to a control group [10]. Also, Mertz et al. [8] and Brown et al. [11-12] found that anodal high voltage pulse current (HVPC) improved epithelialization more rapidly compared with cathodal HVPC.

Our study showed that anodal microamperge DC ES is appropriate for wound closure and return of skin potential to preinjury levels.

\section{CONCLUSIONS}

Our findings demonstrate that endogenous electrical potentials of injured skin play an active role in healing and correlate with WSA. For all the groups, in addition to increased wound closure, the endogenous potentials of the injured skin approached preinjury levels, but by day 21, the electrical potential of the wound site remained positive compared with the surrounding intact skin. Apparently, the use of anodal microamperage DC ES is more appropriate for improving the healing of acute skin wounds because it causes both wounds to close and wound potential to return to preinjury levels faster. We suggest that endogenous potentials of injured skin be studied in infectious and chronic conditions and the effects of DC ES on these endogenous electrical potentials be examined.

\section{ACKNOWLEDGMENTS}

Gadamali Talebi, $\mathrm{PhD}$, is now an assistant professor at Tabriz University of Medical Sciences, Tabiz, Iran.

This material is the result of work supported with resources and the use of facilities at the Tarbiat Modares University, Tehran, Iran.

The authors have declared that no competing interests exist.

\section{REFERENCES}

1. Vodovnik L, Karba R. Treatment of chronic wounds by means of electric and electromagnetic fields. Part 1. Literature review. Med Biol Eng Comput. 1992;30(3):257-66. [PMID: 1453797]

2. Lee RC, Canaday DJ, Doong H. A review of the biophysical basis for the clinical application of electrical fields in soft-tissue repair. J Burn Care Rehabil. 1993;14(3):319-35. [PMID: 8360237]

3. Kloth LC, McCulloch JM. Promotion of wound healing with electrical stimulation. Adv Wound Care. 1996;9(5): 42-45. [PMID: 9069747]

4. Kloth LC. Electrical stimulation for wound healing: A review of evidence from in vitro studies, animal experiments, and clinical trials. Int J Low Extrem Wounds. 2005; 4(1):23-44. [PMID: 15860450]

5. Ennis WJ, Lee C, Meneses P. A biochemical approach to wound healing through the use of modalities. Clin Dermatol. 2007;25(1):63-72. [PMID: 17276203]

6. Athanasiou A, Karkambounas S, Batistatou A, Lykoudis E, Katsaraki A, Kartsiouni T, Papalois A, Evangelou A. The effect of pulsed electromagnetic fields on secondary skin wound healing: An experimental study. Bioelectromagnetics. 2007;28(5):362-68. [PMID: 17486634]

7. Milgram J, Shahar R, Levin-Harrus T, Kass P. The effect of short, high intensity magnetic field pulses on the healing of skin wounds in rats. Bioelectromagnetics. 2004;25(4): 271-77. [PMID: 15114636]

8. Mertz P, Davis S, Cazzaniga A, Cheng K, Reich JD, Eaglstein WH. Electrical stimulation: Acceleration of soft tissue repair by varying the polarity. Wounds. 1993;5(3):153-59.

9. Karba R, Semrov D, Vodovnik L, Benko H, Savrin R. DC electrical stimulation for chronic wound healing enhancement. Part 1. Clinical study and determination of electrical field distribution in the numerical wound model. Bioelectrochem Bioenerg. 1997;43(2):256-70.

10. Alvarez OM, Mertz PM, Smerbeck RV, Eaglstein WH. The healing of superficial skin wounds is stimulated by external electrical current. J Invest Dermatol. 1983;81(2):144-48. [PMID: 6308102]

11. Brown M, Gogia PP. Effects of high voltage stimulation on cutaneous wound healing in rabbits. Phys Ther. 1987;67(5): 662-67. [PMID: 3495010]

12. Brown M, McDonnell MK, Menton DN. Electrical stimulation effects on cutaneous wound healing in rabbits. A followup study. Phys Ther. 1988;68(6):955-60. [PMID: 3259701]

13. Foulds IS, Barker AT. Human skin battery potentials and their possible role in wound healing. Br J Dermatol. 1983; 109(5):515-22. [PMID: 6639877]

14. Barker AT, Jaffe LF, Vanable JW Jr. The glabrous epidermis of cavies contains a powerful battery. Am J Physiol. 1982;242(3):R358-66. [PMID: 7065232]

15. Gentzkow GD. Electrical stimulation to heal dermal wounds. J Dermatol Surg Oncol. 1993;19(8):753-58. [PMID: 8349916]

16. Cunliffe-Barnes T. Healing rate of human skin determined by measurement of electrical potential of experimental abrasions: Study of treatment with petrolatum and with petrolatum containing yeast and liver extracts. Am J Surg. 1945;69: 82-88. 
17. Illingworth CM, Barker AT. Measurement of electrical currents emerging during the regeneration of amputated fingertips in children. Clin Phys Physiol Meas. 1980;1:87-89.

18. Ojingwa JC, Isseroff RR. Electrical stimulation of wound healing. J Invest Dermatol. 2003;121(1):1-12.

[PMID: 12839557]

19. Nuccitelli R. A role for endogenous electric fields in wound healing. Curr Top Dev Biol. 2003;58:1-26. [PMID: 14711011]

20. Hampton S, King L. Healing an intractable wound using bioelectrical stimulation therapy. Br J Nurs. 2005;14(15 Suppl): S30-32. [PMID: 16116416]

21. Christie MJ, Venables PH. Sodium and potassium electrolytes and "basal" skin potential levels in male and female subjects. Jpn J Physiol. 1971;21(6):659-68. [PMID: 5317181]

22. Christie MJ, Venables PH. Characteristics of palmar skin potential and conductance in relaxed human subjects. Psychophysiology. 1971;8(4):525-32. [PMID: 5094935]

23. Watson T. The bioelectric correlates of musculoskeletal injury and repair [dissertation]. Surrey (England): University of Surrey; 1994.

24. Jerinovi A, Bobanovic F, Vodovnik L. Endogenous potentials in two different models of human skin injuries. Bioelectrochem Bioenerg. 1993;30:221-27.

25. Friedenberg ZB, Brighton CT. Bioelectric potentials in bone. J Bone Joint Surg Am. 1966;48(5):915-23. [PMID: 5942807]

26. Wilber MC. Surface direct current bioelectric potentials in the normal and injured human thigh. Tex Rep Biol Med. 1978;36:197-204. [PMID: 725792]
27. Brown M, Gogia PP, Sinacore DR, Menton DN. Highvoltage galvanic stimulation on wound healing in guinea pigs: Longer-term effects. Arch Phys Med Rehabil. 1995;76(12): 1134-37. [PMID: 8540790]

28. Takan I, Ozyanzgan I, Tercan M, Karda HY, Balkanli S, Saraymen R, Zorlu U, Ozügül Y. A comparative study of the effect of ultrasound and electrostimulation on wound healing in rats. Plast Reconstr Surg. 1997;100(4):966-72. [PMID: 9290665]

29. Reger SI, Hyodo A, Negami S, Kambic HE, Sahgal V. Experimental wound healing with electrical stimulation. Artif Organs. 1999;23(5):460-62. [PMID: 10378943]

30. Burr HS, Harvey SC, Taffel M. Bio-electric correlate of wound healing. Yale J Biol Med. 1940;2:103-7.

31. Becker RO. The sign of the miracle. In: Becker RO, Selden $\mathrm{G}$, editors. The body electric: Electromagnetism and the foundation of life. New York: Morrow; 1985. p. 72.

32. Chang KS, Snellen JW. Bioelectric activity in the rabbit ear regeneration. J Exp Zool. 1982;221(2):193-203.

[PMID: 7097182]

33. Edelberg R. Relation of electrical properties of skin to structure and physiologic state. J Invest Dermatol. 1977;69(3): 324-27. [PMID: 894073]

34. Jaffe LF, Vanable JW Jr. Electric fields and wound healing. Clin Dermatol. 1984;2(3):34-44. [PMID: 6336255]

Submitted for publication May 15, 2007. Accepted in revised form September 17, 2007. 